\title{
The Phase of Thalamic Alpha Activity Modulates Cortical Gamma-Band Activity: Evidence from Resting-State MEG Recordings
}

\author{
Frédéric Roux, ${ }^{1,2}$ Michael Wibral, ${ }^{2}$ Wolf Singer, ${ }^{3,4,5}$ Jaan Aru, ${ }^{3,5}$ and Peter J. Uhlhaas ${ }^{3,4,6}$ \\ ${ }^{1}$ Basque Center on Cognition, Brain, and Language (BCBL), 2009 Donostia/San Sebastian, Spain, ${ }^{2}$ MEG Unit, Brain Imaging Center, Goethe University, \\ 60528 Frankfurt am Main, Germany, ${ }^{3}$ Max-Planck Institute for Brain Research, Department of Neurophysiology, 60438 Frankfurt am Main, Germany, \\ ${ }^{4}$ Ernst Strüngmann Institute, 60528 Frankfurt am Main, Germany, ${ }^{5}$ Frankfurt Institute for Advanced Sciences, 60438 Frankfurt am Main, Germany, and \\ ${ }^{6}$ Institute of Neuroscience and Psychology, Glasgow University, Glasgow, G12 8QB Scotland
}

Recent findings have implicated thalamic alpha oscillations in the phasic modulation of cortical activity. However, the precise relationship between thalamic alpha oscillations and neocortical activity remains unclear. Here we show in a large sample of healthy human participants $(n=45)$ using spatial filtering techniques and measures of phase amplitude coupling that the amplitude of gamma-band activity in posterior medial parietal cortex is modulated by the phase of thalamic alpha oscillations during eyes-closed resting-state recordings. In addition, our findings show that gamma-band activity in visual cortex was not modulated by thalamic alpha oscillations but coupled to the phase of strong cortical alpha activity. To overcome the limitations of electromagnetic source localization we estimated conduction delays using transfer entropy and found nonspurious information transfer from thalamus to cortex. The present findings provide novel evidence for magneto-encephalography-measured phase coupling between cortical gamma-band activity and thalamic alpha oscillations, which highlight the role of phasic inhibition in the coordination of cortical activity.

\section{Introduction}

A growing body of evidence implicates rhythmic activity in the alpha band $(8-13 \mathrm{~Hz})$ as a core feature of cortical communication (Jensen and Mazaheri, 2010) and cognition (VanRullen and Koch, 2003). This is supported by evidence demonstrating that the phase of the alpha cycle modulates neural firing (Haegens et al., 2011), perceptual detection rates (Varela et al., 1981; Dugué et al., 2011), and cerebral blood flow (Scheeringa et al., 2011), suggesting that alpha-band oscillations exert critical influence on the excitability level of neocortical networks as well as on the timing of neuronal responses (Klimesch et al., 2007; Jensen and Mazaheri, 2010).

One brain region that could be crucially involved in this process is the thalamus, a key structure involved in the generation of alpha oscillations (Andersen and Andersson, 1968; da Silva et al., 1973, Lopes da Silva, 1980; Jahnsen and Llinás, 1984; Hughes and Crunelli, 2005; Bollimunta et al., 2011). Recent data show that alpha-band rhythms generated in the thalamus are linked with the temporal

Received Dec. 17, 2012; revised Sept. 27, 2013; accepted 0ct. 6, 2013.

Author contributions: F.R. and P.J.U. designed research; F.R. performed research; F.R. and M.W. contributed unpublished reagents/analytic tools; F.R. analyzed data; F.R., M.W., W.S., J.A., and P.J.U. wrote the paper.

This work was supported by the Max-Planck society, the LOEWE Grant "Neuronale Koordination Forschungsschwerpunkt Frankfurt," and the Basque Center on Cognition, Brain, and Language.

The authors declare no competing financial interests.

This article is freely available online through the $J$ Neurosci Author Open Choice option.

Correspondence should be addressed to Dr. Peter J. Uhlhaas, Institute of Neuroscience and Psychology, University

of Glasgow, Hillead Street 58, Glasgow, Scotland G12 8QB. E-mail: peter.uhlhaas@glasgow.ac.uk.

DOI:10.1523/JNEUROSCI.5778-12.2013

Copyright $\odot 2013$ the authors $\quad 0270-6474 / 13 / 3317827-09 \$ 15.00 / 0$ coordination of spiking activity in thalamo-cortical (TC) circuits (Lorincz et al., 2009; Vijayan and Kopell, 2012), providing important support for the inhibition-timing hypothesis of alpha oscillations (Klimesch et al., 2007; Jensen and Mazaheri, 2010).

Moreover there is emerging evidence that information transfer from thalamic nuclei, such as the pulvinar or the lateral geniculate nucleus (LGN), to cortex is mediated via TC synchronization in the alpha band (Lorincz et al., 2009; Saalmann et al., 2012), raising the possibility that coherent TC alpha oscillations may contribute to the modulation of cortical gamma-band activity. However, evidence for this relationship is so far lacking.

To investigate this question, we recorded resting-state activity in a large sample $(N=45)$ of healthy participants with magnetoencephalography (MEG). We focused on interactions between alpha $(8-13 \mathrm{~Hz})$ and broadband gamma $(30-70 \mathrm{~Hz})$ activity as previous work indicates that broadband activity in the gamma frequency range reflects active neuronal processing such as neuronal spiking activity (Rasch et al., 2008; Manning et al., 2009; Whittingstall and Logothetis, 2009; Miller, 2010; Buzsáki et al., 2012) and is modulated by the phase of task related and spontaneous alpha oscillations (Chorlian et al., 2006; Osipova et al., 2008; Cohen et al., 2009; Voytek et al., 2010; Foster and Parvizi, 2012; Saalmann et al., 2012; Spaak et al., 2012; Yanagisawa et al., 2012). Using a whole brain analysis as well as a seed region-based analysis of phase-amplitude coupling (PAC) at source level, we examined the modulation of neocortical gamma-band activity by the phase of cortical and thalamic alpha oscillations. Finally, we tested the physiological validity of our findings using transfer entropy (TE) and addressed the potential contribution of spatial 
source leakage using a reversed-seed region analysis to control for spurious TC interactions.

\section{Materials and Methods}

MEG data acquisition and preprocessing. Four minutes of eyes-closed spontaneous MEG activity were recorded (sampling rate: $1.2 \mathrm{kHz}$, lowpass filter: $600 \mathrm{~Hz}$ ) using a whole-head MEG system with 275 axial gradiometers (Omega 2005; VSM MedTech) from $n=45$ healthy adult participants, which were aged between 18 and 27 years old (mean age: 20.5 years; SD: 2; 24 females). The study was approved by the ethics committee of the Goethe University Frankfurt, and written informed consent was obtained from participants before the recording. The verti$\mathrm{cal}$ and horizontal electro-oculograms were recorded to monitor eye movements for artifacts. Partial artifact rejection was performed by rejecting segments of the trials containing eye-blinks, muscle, and SQUID artifacts using an automatic artifact rejection algorithm as described by Oostenveld et al. (2011). On average, $163 \pm 53$ s of artifact-free data underwent subsequent analysis. In addition, independent component analysis (Bell and Sejnowski, 1995) was used to remove cardiac activity as well as residual eye movements.

Structural magnetic resonance imaging data acquisition. Structural magnetic resonance images (MRIs) were obtained with a $3 \mathrm{~T}$ Siemens Allegra scanner device (Siemens) using the standard CP birdcage headcoil and a T1 sequence. Eleven participants met an exclusion criterion for MRI scans and were not included for source analyses.

\section{Data analysis}

Source localization. A linearly constrained minimum variance beamformer algorithm (Sekihara et al., 2004) was used to generate maps of source activity in a spatial grid of 4590 points with a resolution of $1 \mathrm{~cm}$. Coregistration with Montreal Neurological Institute (MNI) coordinates was based on three fiducial coils placed on the nasion and the left and right pre-auricular ridges. The forward solution for each participant was estimated from individual head models using a common dipole grid in MNI space, which was warped onto the anatomy of each participant and a realistic single-shell volume conductor model (Nolte, 2003). Virtual electrodes were estimated for each grid location by calculating the beamformer weights from the sensor covariance matrix and multiplying the bandpass filtered sensor signals with the beamformer weights.

Spectral analysis. Spectral power was estimated for MEG signals at sensor level at frequencies between 1 and $100 \mathrm{~Hz}$, using multitaper spectral analysis (Percival and Walden, 1998). The spectrogram for MEG sensor signals was computed from windows of $10 \mathrm{~s}$, which were displaced in time at $1 \mathrm{~s}$ steps and a spectral concentration of $1.5 \mathrm{~Hz}$ per frequency bin using 29 Slepian tapers.

\section{Cross-frequency coupling}

Alpha-gamma PAC. PAC was estimated following previously described approaches (Sauseng et al., 2009; Miller et al., 2010). In brief, a phase time series $\varphi(t)$ is estimated for every sample of an MEG sensor or source time series $x(t)$ using Morlet wavelets (Tallon-Baudry and Bertrand, 1999) and $\varphi(t)$ is sorted into values ranging from $-\pi$ to $\pi(\mathrm{rad})$. We estimated $\varphi(t)$ for frequencies ranging from 1 to $15 \mathrm{~Hz}$ to be able to detect possible PAC effects occurring between gamma-band amplitude and slower rhythms. The amplitude of gamma-band activity $\gamma(t)$ was obtained by first bandpass filtering $x(t)$ between 30 and $70 \mathrm{~Hz}$ using a fourth order Butterworth filter. Next we applied the Hilbert transform to $x(t)$ to obtain a complex time series $h(t)$, and $\gamma(t)$ was estimated from the absolute value of $h(t)$. Finally, $\gamma(t)$ was log transformed before the $z$-score was computed by demeaning and dividing $\gamma(t)$ by the SD. To prevent artifacts arising from aliasing, the cutoff for the upper limit of frequencies at which $\varphi(t)$ was estimated was set to $15 \mathrm{~Hz}$, so that the upper frequency limit used to estimate $\varphi(t)$ and the lower frequency bound used for the estimation of $\gamma(t)$ were separated by a factor of two. Finally, the samples of $\gamma(t)$ were aligned in time with the samples of the sorted phase time series $\varphi(t)$ and averaged over 51 equally wide phase bins. This resulted in a phase-amplitude distribution for every frequency between 1 and $15 \mathrm{~Hz}$.

Modulation index. To map the alpha-gamma PAC in source space we computed the Modulation Index (MI) from alpha-gamma phase- amplitude distributions computed from virtual channel activity. The MI is an adaptation of the Kullback-Leibler divergence (Kullback and Leibler, 1951) and measures the distance of an empirical amplitude distribution over phase bins from the uniform distribution. The assumption underlying this metric is that MI values reflect the degree of PAC between two frequency bands. Therefore, grid points with high MI values reflect a higher degree of PAC as compared with grid points with lower MI values (see Tort et al., 2010 for more details).

\section{TC information transfer}

TC alpha-gamma PAC. Similarly, TC alpha-gamma coupling was estimated by computing phase time series at alpha frequencies $(8-13 \mathrm{~Hz})$ using spatial filters located in the left $(x=-10, y=-20, z=10)$ and right $(x=10, y=-20, z=10)$ thalamus. Our approach is thus similar to the methodology used by previous MEG studies, which have successfully measured TC synchronization with a sensitivity of $2 \mathrm{~cm}$ around thalamic regions (Tarkiainen et al., 2003; Lou et al., 2010). MI values were then calculated from the histogram of gamma-band amplitude with respect to the thalamic alpha phase for each point within the grid.

TE. Spurious TC alpha-gamma coupling in MEG data can hypothetically arise from an interaction that is exclusively corticothalamic (CT) in nature or from volume conduction effects where cortical alpha oscillations are mistaken for thalamic sources. To rule out the first possibility, it is necessary to test for directed information transfer from thalamus to cortex, while a test for the presence of significant volume conduction effects is needed to control for the second scenario. TE analysis is sensitive to linear and nonlinear interactions such as cross-frequency couplings (Vicente et al., 2011) and allows the detection of volume conduction effects (Lindner et al., 2011). Moreover, the interaction delay underlying the information transfer can be reconstructed using a delaysensitive TE estimator as shown recently by our group (Wibral et al., 2013). Together, this allows us to test and rule out the possibilities of a purely CT coupling and volume conduction artifacts as the cause of the observed TC interaction. Furthermore, a comparison of the reconstructed interaction delays from thalamus to cortex in our data with values reported in the literature can be used to cross-validate our results in terms of physiological plausibility. In this study, delay-sensitive TE was computed as follows:

$$
T E(X \rightarrow Y, u)=I\left(Y_{t} ; \mathbf{X}_{\mathbf{t}-\mathbf{u}} \mid \mathbf{Y}_{\mathbf{t}-\mathbf{1}}\right),
$$

where $X$ and $Y$ are two discrete-time random processes with a state-space representation $X, Y$, and where $u$ is the assumed interaction delay from $X$ to $Y$. Here, $I\left(Y_{t} ; \mathbf{X}_{\mathbf{t}-\mathbf{u}} \mid \mathbf{Y}_{\mathbf{t}-1}\right)$ stands for the mutual information between the past state of the source process, $\mathbf{X}_{\mathbf{t}-\mathbf{u}}$, the current value of the target process, $Y_{t}$, conditional on the past state of the target, $\mathbf{Y}_{\mathbf{t}-\mathbf{1}}$. The true interaction delay $\delta$ is found by maximizing $T E(X \rightarrow Y, u)$ with respect to $u$ :

$$
\delta=\arg \max _{\mathrm{u}}(T E(X \rightarrow Y, u))
$$

State-space reconstruction parameters were optimized using Ragwitz' criterion to ensure optimal self prediction of the target process as requested by Wiener's principle (Ragwitz and Kantz, 2002). The KraskovStögbauer-Grassberger (Kraskov et al., 2004) estimator was used in combination with nonparametric statistical testing against epochshuffled surrogate data as detailed in Lindner et al. (2011). Furthermore, TE was computed from thalamus to posterior medial parietal cortex with individual embedding parameters for each participant and connection.

TC phase synchronization. Phase synchronization between thalamus and cortex was measured by means of the phase locking value (PLV) (Lachaux et al., 1999) for phase time series obtained using Morlet wavelets for frequencies between 1 and $100 \mathrm{~Hz}$.

\section{Statistical analysis}

Statistical significance at sensor level. At MEG sensor level, the statistical significance of the estimated PAC was assessed by randomly shifting the phase time series in time by at least 5001 points with respect to the time course of gamma-band amplitude for every MEG channel and frequency between 1 and $15 \mathrm{~Hz}$. The mean average gamma-band amplitude was 
then recalculated as a function of phase for the shifted amplitude time series. This procedure was repeated 200 times to obtain the average shifted PAC. A nonparametric permutation-based Monte Carlo approach was used to estimate the significance of the empirical PAC between cortical rhythms in the $1-15 \mathrm{~Hz}$ range and gamma-band amplitude as compared with the average shifted data. In short, this approach consists in calculating dependent $t$ values for each frequency and phase bin and every channel to measure the consistency of the difference between the empirical and shifted data across participants and comparing these $t$ values to a distribution of $t$ values calculated from randomly recomposed empirical and shifted datasets. A cluster-algorithm-based approach was then used to correct for multiple comparisons (Maris and Oostenveld, 2007). The threshold for the clustering entry level and for statistical significance of the clusters was $p<0.01$ (corrected).

Statistical significance of PAC at source level. To assess the statistical significance of alpha-gamma coupling at source level we compared empirical MI values to MI values estimated from shifted data using the same permutation-based Monte Carlo approach that was used to assess the statistical significance of alpha-gamma PAC at sensor level. At the source level dependent $t$ values were calculated to contrast the MI values estimated from empirical data against MI values estimated from shifted data for each grid point in source space. These $t$ values hence measure the consistency of the difference between empirical and shifted MI values across participants. The threshold for the clustering entry level and statistical significance of the clusters was $p<0.01$ (corrected).

Statistical significance of TE. We used permutation testing $\left(n=5^{\star} 10^{6}\right.$ permutations) (Lindner et al., 2011) to compare the empirical TE values against values obtained from surrogate datasets. Surrogate data were generated by shifting the source time series (thalamus) with respect to the target time series (cortex). For each participant, results where thresholded at a level of $p<0.05$ (uncorrected). Second level group level statistics for the presence of links were then performed using one-sided binomial tests (binomial distribution) with a significance level of $p<0.05$ (Bonferroni corrected). In addition, we performed a shift test to reject false positive TE due to volume conduction effects (Lindner et al., 2011).

\section{Analysis software}

All analyses were performed using custom MATLAB scripts and open source MATLAB toolboxes as well as FSLview (fieldtrip: http://fieldtrip. fcdonders.nl/; chronux: http://www.chronux.org/; SPM2: http://www. fil.ion.ucl.ac.uk/spm/; TRENTOOL: http://www.trentool.de/; FSLview: http://fsl.fmrib.ox.ac.uk/fsl/fslview/).

\section{Results}

\section{Spectral estimates at sensor level}

We observed a peak of spectral power at $\sim 10 \mathrm{~Hz}$ in the grand average power spectrum as well as in the distribution of peak frequencies (Fig. 1a,b). In addition, we found a negative correlation between spectral power in the $8-13 \mathrm{~Hz}$ and $30-70 \mathrm{~Hz}$ frequency ranges, which was significant in 36 of $45(80 \%)$ participants (mean Pearson correlation coefficient -0.38 ; $\mathrm{SD}=$ $0.24 ; p<0.05 ; p<0.0001$; binomial test; two-sided; Fig. $1 c, d$ ).

\section{PAC at sensor level}

Time-frequency representations of epochs, which were phase aligned with the occipital alpha rhythm, revealed a strong phasic entrainment of spectral power in the $30-70 \mathrm{~Hz}$ frequency range (Fig. $2 a$ ). This was confirmed by the topography of statistical $t$ values, which revealed two peaks of significant PAC over left and right occipital channels (Fig. 2b). In addition, phase-frequency representations of statistical $t$ values revealed that the amplitude of $30-70 \mathrm{~Hz}$ activity was significantly modulated by the phase of frequencies in the alpha range $(8-13 \mathrm{~Hz})$ and that gamma-band activity was either in phase or anti-phase with the alpha cycle over left or right sensors, respectively ( $p<0.01 ; t$ test; two-sided; corrected; Fig. $2 c, d)$.
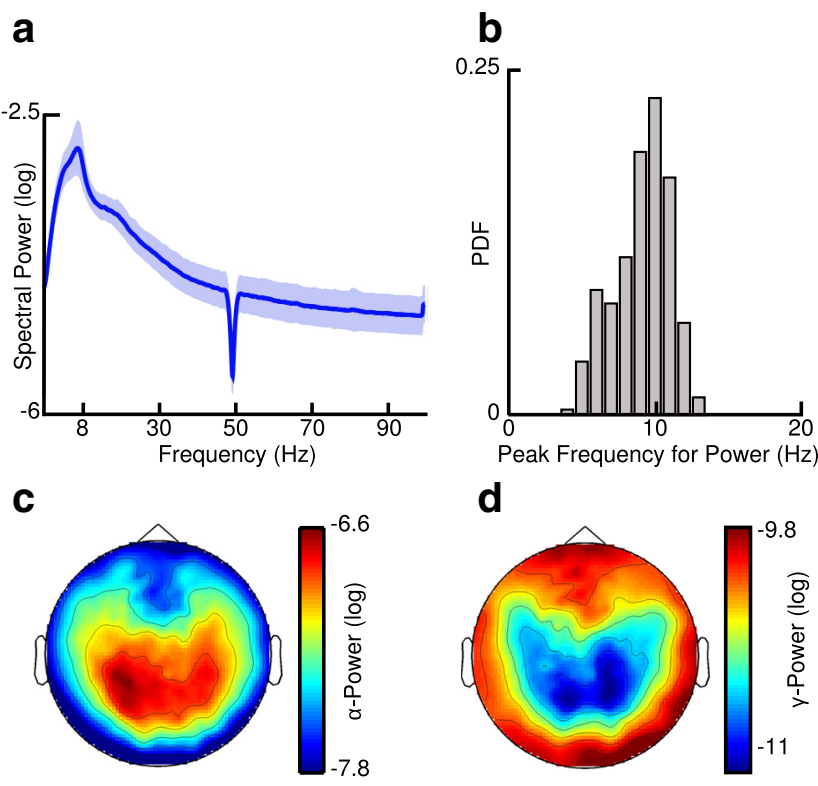

Figure 1. Spectral power at sensor level. $\boldsymbol{a}, \boldsymbol{b}$, Grand average power spectrum $(\boldsymbol{a})$ of resting state activity and probability density function (PDF) of peak frequencies (b) showing strong activity in the alpha band. $\boldsymbol{c}, \boldsymbol{d}$, Topographies of log transformed (c) alpha-band $(8-13 \mathrm{~Hz})$ and $(\boldsymbol{d})$ gamma-band $(30-70 \mathrm{~Hz})$ power.

\section{a}
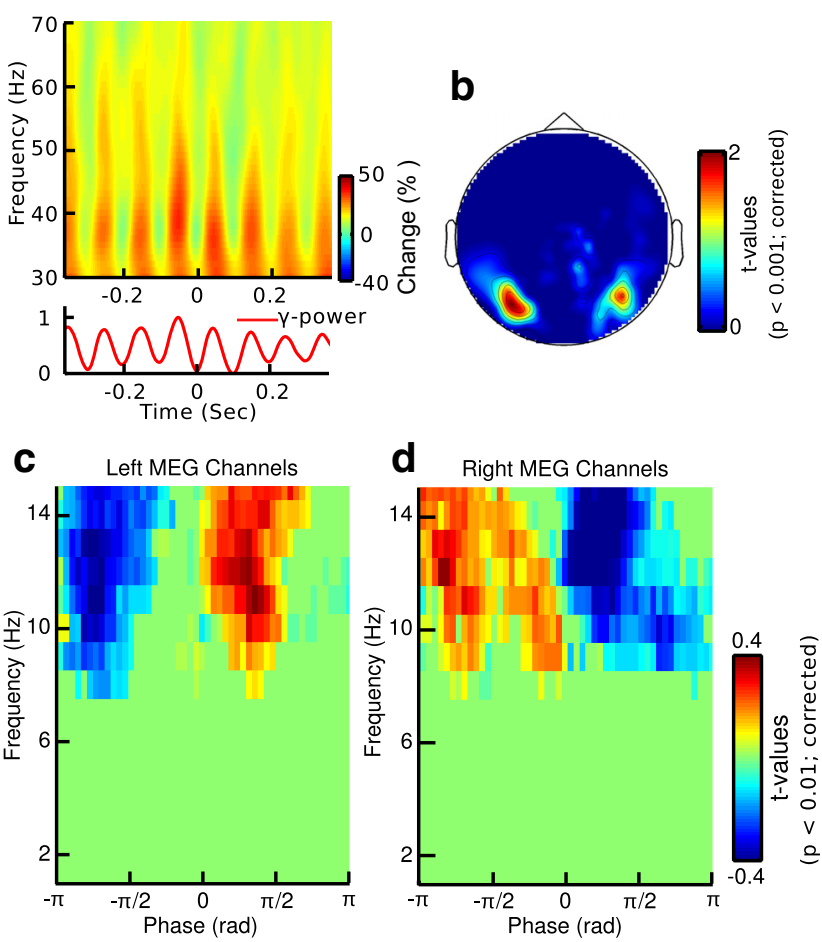

Figure 2. Alpha-gamma PAC at sensor level. $\boldsymbol{a}$, Time-frequency map of MEG signals phase aligned with the peak of alpha activity in one subject. $\boldsymbol{b}$, Topography of statistical $t$ values averaged over significant frequencies ( $p<0.01$; dependent $t$ test; two-sided; corrected) for the comparison of alpha-gamma PAC with shifted data at sensor level (axial gradients). $\boldsymbol{c}, \boldsymbol{d}$, Average phase-frequency maps of statistical $t$ values for MEG sensors showing significant PAC for different alpha phases. The left versus right pattern arises from the bipolar pattern of a magnetic field as measured by the axial gradiometers. The pseudo colors indicate increased (warm colors) and decreased (cold colors) gamma-band activity as compared with shifted data. 

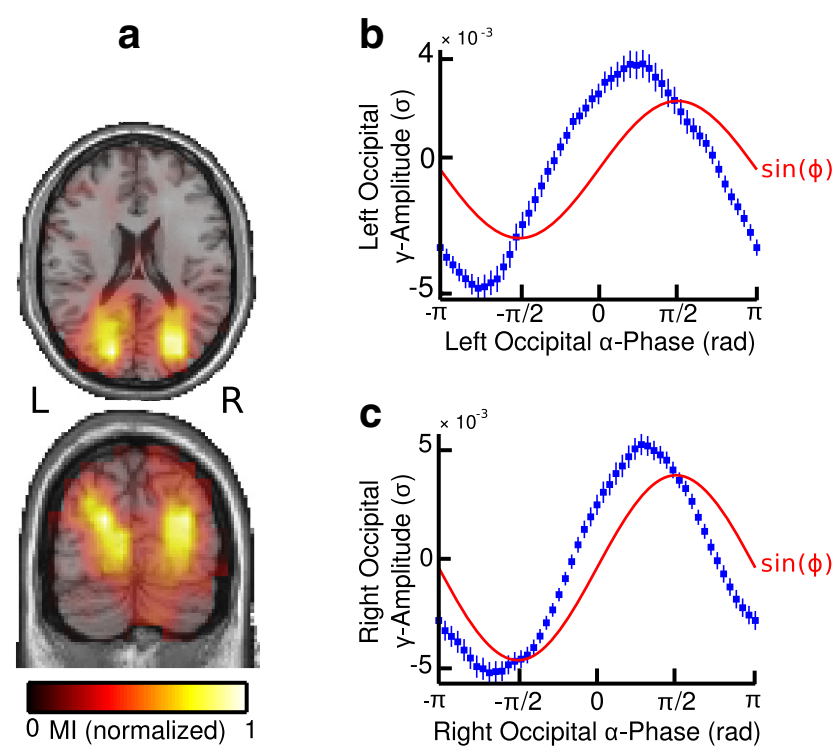

Figure 3. Alpha-gamma PAC at source level. $\boldsymbol{a}$, Source maps of normalized MI values displayed on axial and coronal sections of the MNI template brain. The map shows two clusters of MI values in the left and right visual cortex, indicating enhanced local PAC in the visual cortex. $\boldsymbol{b}$, c, Cortical gamma-band amplitude as a function of local alpha phase averaged over grid points in the left $(\boldsymbol{b})$ and right (c) visual cortex. Error bars indicate SEM; red line represents the fit of a sinusoidal function $(\sin (\varphi))$ to the data. R, right; L, left.

Table 1. MNI coordinates for local PAC

\begin{tabular}{|c|c|c|c|}
\hline \multirow[b]{2}{*}{ Anatomical label } & \multicolumn{3}{|c|}{ MNI coordinates } \\
\hline & $x$ & $y$ & $z$ \\
\hline Left BA 18 & -24 & -78 & -4 \\
\hline Right BA 18 & 16 & -76 & -4 \\
\hline Left cuneus & -24 & -74 & 8 \\
\hline Right cuneus & 14 & -76 & 8 \\
\hline
\end{tabular}

BA, Brodmann area.

\section{Cortical sources of alpha-gamma PAC}

Similarly to our results at sensor level, we observed two spatially separated clusters of MI values in the left and right occipital regions comprising Brodmann area 18 and the cuneus (Fig. $3 a$, Table 1). The peak of the histogram of mean gamma-band amplitude values over local alpha phase bins was shifted toward the descending phase of the alpha cycle $(\pi / 2 \mathrm{rad})$ and there was a trend toward a positive correlation between the phase bins at which occipital gamma-band amplitudes were highest across both hemispheres $(r=0.29$; $p=0.06$; Fisher's correlation coefficient for circular data; Fig. $3 b, c$ ).

\section{Thalamic sources of alpha-gamma PAC}

Based on prior findings, which have highlighted the importance of thalamic oscillations for the phasic control of spiking activity in TC circuits (Lorincz et al., 2009; Vijayan and Kopell, 2012), we estimated the phase of thalamic alpha activity from two virtual electrodes in the thalamus and examined the coupling of brain-wide gamma-band activity to the phase of the thalamic alpha rhythm. This approach revealed that TC alpha-gamma PAC was significant in posterior medial parietal areas, comprising the left and right precuneus as well as the right posterior cingulate area $(p<0.01 ; t$ test; two-sided; corrected; Fig. $4 a, b$, Table 2). Moreover, the histogram of mean gammaband amplitude values was centered at the peak of the thalamic alpha phase $(0 \mathrm{rad})$, and there was a positive and significant correlation between phase bins at which cortical gamma-band activity reached maxima in both hemispheres $(r=0.36$; $p<0.05$; Fisher's correlation coefficient for circular data; Fig. $4 c, d)$. Furthermore, alpha power in the posterior medial parietal cortex was greatly reduced as compared with alpha power in the visual cortex $\left(t_{(34)}=7.98\right.$; $p<0.001$; twosided $t$ test).

\section{TC information transfer}

We observed that phase synchronization between the thalamus and the cortical regions, which showed significant coupling with the phase of thalamic alpha activity was particularly enhanced at alpha frequencies (Fig. 4e). Furthermore, we estimated conduction latencies from thalamus to cortex using TE analysis and found nonspurious TC information transfer as well as physiologically realistic conduction delays (range: 12.5-19.8 ms; mean: $15.8 \mathrm{~ms}$; SD: $2.4 \mathrm{~ms}$; binomial test, $p<0.01$; FDR corrected; Fig. $4 f$ ). Accordingly, these findings suggest a directed information transfer from thalamic source locations to posterior medial parietal cortex as opposed to nonspecific source activity resulting from spatial leakage.

\section{Cross-validation of thalamic source estimates and reversed-seed region analyses}

The aim of the seed-region analysis was to detect a modulatory relationship between the phase of thalamic alpha oscillations and the amplitude of cortical gamma-band activity. However, because of the limited spatial resolution of MEG beamformers, the possibility that activity from other nonthalamic sources leaked to thalamic source positions could not be excluded. As our seedregion analysis only focused on the thalamus, our approach may have lead to a false detection of TC alpha-gamma coupling due to activity from nonthalamic sources.

To examine whether potential alpha leakage from nonthalamic sources may have biased our results, we conducted a reversed-seed analysis and quantified source leakage through estimating the modulation of gamma-band activity in posterior medial parietal cortex by the phase of alpha activity for each grid point in source space. We hypothesized that if thalamic alpha activity would be contaminated by activity from nonthalamic sources, alpha-gamma PAC would occur in voxels located outside of the thalamus and the posterior medial parietal cortex.

The results of this reversed-seed region analysis revealed a strong modulation of gamma-band activity by the phase of alpha oscillations in the posterior medial parietal cortex as well as in the left thalamus (Fig. 5a, Table 3). In addition, we observed that the histograms of mean gamma-band amplitude values were centered at the peak of the alpha phase $(0 \mathrm{rad})$ in both the parietal areas and the left thalamus (Fig. $5 b$ ), which are similar to the results when the thalamus was used as a seed (Fig. $4 c, d$ ).

\section{Spatial leakage}

To assess the influence of spatial leakage, we measured the extent to which spatial filters in the thalamus may have been contaminated by activity from nonthalamic sources by measuring the correlation between the spatial filters used to estimate thalamic activity and the spatial filters used to estimate source activity at each grid point in source space. This approach allowed us to quantify to which extent the spatial filters used to reconstruct activity in the thalamus were influenced by activity coming from other potential nonthalamic sources of alpha activity. We found that overall there was a weak correlation between thalamic and nonthalamic spatial filters (left hemisphere: $r=0.03, \mathrm{SD}=0.02, p=0.21, \mathrm{SD}=0.03$; right hemisphere: $r=0.04 ; \mathrm{SD}=0.02, p=0.19, \mathrm{SD}=0.02$ ) and that spatial filters in the thalamus were only weakly correlated with spatial filters 


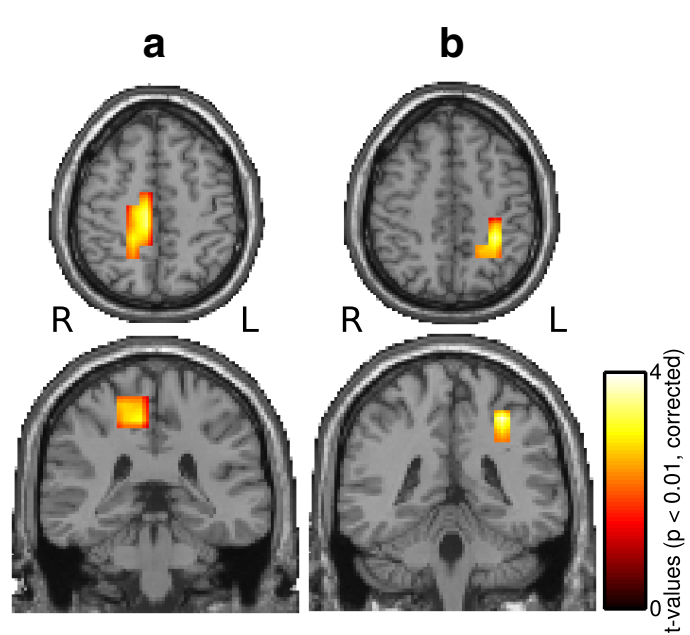

Figure 4. TC-PAC. $\boldsymbol{a}, \boldsymbol{b}$, Statistical source maps of $t$ values ( $p<0.01$; dependent $t$ test; two-sided; corrected) for the comparison of TC alpha-gamma PAC with shifted data for seed regions in the left $(\boldsymbol{a})$ and right ( $\boldsymbol{b}$ ) thalamus. $\boldsymbol{c}, \boldsymbol{d}$, Cortical gamma-band amplitude as a function of thalamic alpha phase averaged over grid points in the left (c) and right (d) posterior medial parietal cortex. Error bars indicate SEM, and the red line represent the fit of a cosine function $(\cos (\varphi))$ to the data. $\boldsymbol{e}$, Phase synchronization between the thalamus and posterior medial parietal areas in the left (black) and right (red) hemisphere for frequencies from 1 to $100 \mathrm{~Hz}$. Phase synchronization was measured by means of the PLV.f, Probability density function (PDF) of TC conduction delays as measured by TE (mean: $15.8 \mathrm{~ms} ; \mathrm{SD}: 2.4 \mathrm{~ms}$ ). R, right; L, left.

Table 2. MNI coordinates for TC-PAC

\begin{tabular}{|c|c|c|c|}
\hline \multirow[b]{2}{*}{ Anatomical label } & \multicolumn{3}{|c|}{ MNI coordinates } \\
\hline & $x$ & $y$ & $z$ \\
\hline Left precuneus & -24 & -50 & 48 \\
\hline Right precuneus & 18 & -46 & 50 \\
\hline Left intraparietal sulcus & -30 & -52 & 36 \\
\hline Right posterior cingulate cortex & 8 & -28 & 50 \\
\hline
\end{tabular}

in posterior medial parietal cortex (right parietal cortex: $r=0.003$, $\mathrm{SD}=0.1, p=0.22, \mathrm{SD}=0.11$; left parietal cortex: $r=0.02, \mathrm{SD}=$ $0.06, p=0.2, \mathrm{SD}=0.17$; Fig. 6 ).

\section{Discussion}

The goal of the present study was to examine the relationship between thalamic alpha oscillations and neocortical gamma-band activity in resting-state MEG recordings. The thalamus has been involved in the regulation of information transmission to the cortex via the modulation of response magnitude, firing mode, and synchrony of neurons (Saalmann and Kastner, 2009). Alpha oscillations are a prominent feature of electrophysiological resting state recordings in humans (Berger, 1929; Adrian and Matthews, 1934) and have been linked with phasic inhibition (Klimesch et al., 2007; Osipova et al., 2008; Jensen and Mazaheri, 2010; Dugué et al., 2011; Scheeringa et al., 2011), which could be an effective mechanism to regulate the output of cortical networks (Jensen et al., 2012). However, it remains currently unclear if thalamic alpha oscillations could modulate cortical gamma-band activity.

Consistent with previous work (Chorlian et al., 2006; Osipova et al., 2008; Cohen et al., 2009; Voytek et al., 2010; Foster and Parvizi, 2012; Saalmann et al., 2012; Spaak et al., 2012; Yanag- isawa et al., 2012), we observed that local gamma-band activity was coupled to the phase of the alpha-band rhythm, which was most pronounced over the visual cortex, suggesting that fluctuations of broadband gamma-band activity are linked to the phase of the alpha cycle. Moreover, source-space wide testing of PAC revealed that fluctuations of gamma-band activity in the visual cortex were greatest near the trough of local alpha activity.

In contrast, TC alpha-gamma coupling became significant only in posterior medial parietal cortex where cortical gamma-band activity was greatest at the peak of the thalamic alpha rhythm. Recent findings from intracranial recordings in nonhuman primates suggest that gamma-band activity is differentially entrained by the phase of independent alpha oscillations in granular and infragranular layers (Spaak et al., 2012), which are characterized by a polarity inversion across layers in the current source density profile (Bollimunta et al., 2008; Spaak et al., 2012). From this perspective, layer-dependent phase relationships may account for the differential modulation of gamma-band activity in the visual cortex and the posterior medial parietal cortex in the present study.

Importantly, TE analysis revealed significant and directed information transfer from the thalamus to posterior medial parietal cortex with TC conduction latencies of 12.5-19.8 ms, which are in agreement with previously reported TC delays (Lopes da Silva et al., 1980). Specifically, Lopes da Silva et al. (1980) have reported conduction delays between the pulvinar and the visual cortex, which were $\sim 15-21 \mathrm{~ms}$. Thus, the results of the TE analysis suggest that the TC alpha-gamma coupling could reflect physiological meaningful information transfer and not simple volume conduction.

To further substantiate this possibility, we used a reversedseed region analysis and tested our spatial filters for signal leak- 


\section{Reversed Seed-Region Analysis}
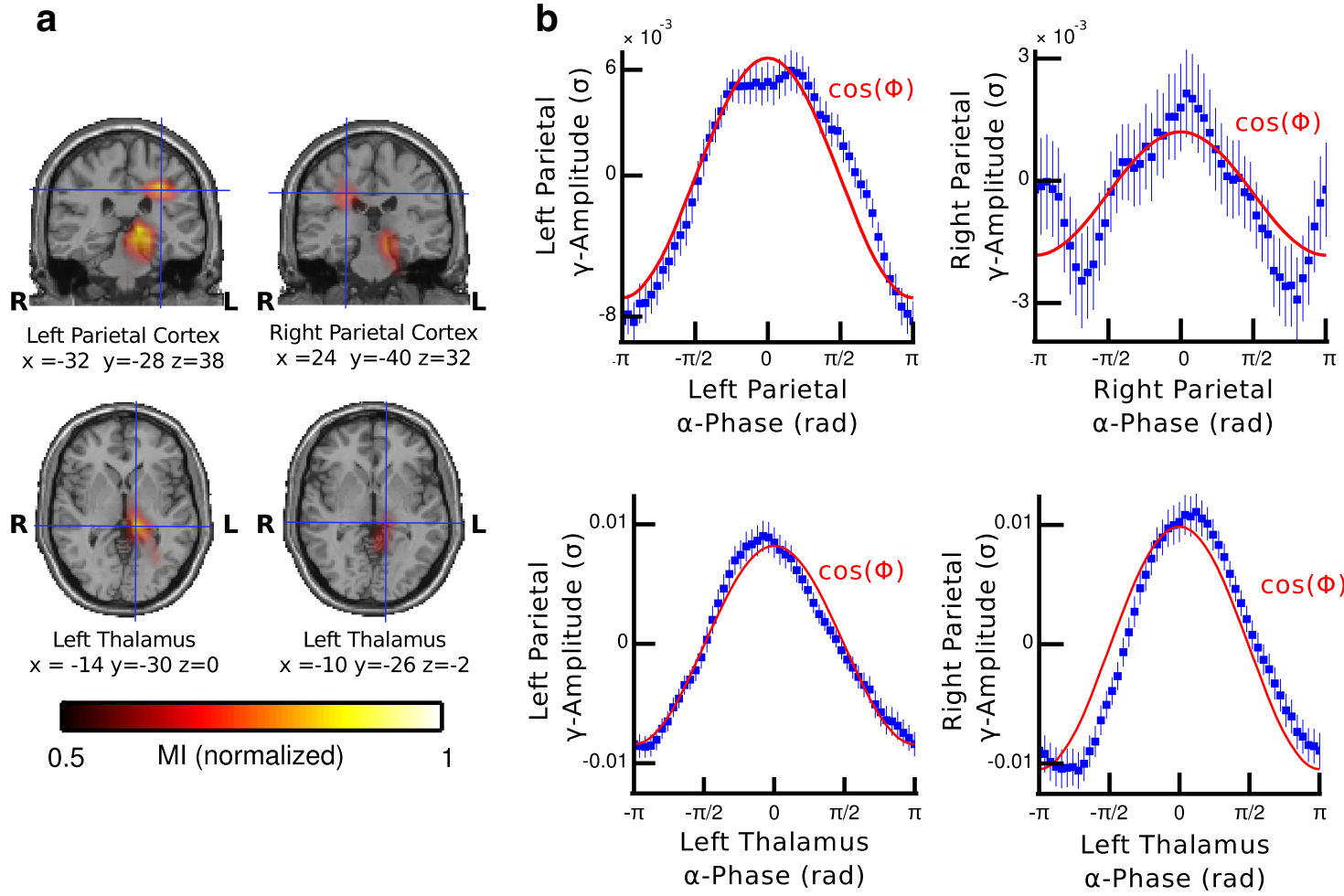

Figure 5. Reversed-seed region analysis. $\boldsymbol{a}$, Source maps of normalized Ml values for the modulation of $30-70 \mathrm{~Hz}$ activity in the left and right posterior-medial parietal cortex by the phase of brain-wide alpha oscillations. The amplitude of broadband gamma activity in posterior-medial parietal cortex was modulated by the phase of alpha oscillations in the left thalamus as well as in the left and right posterior-medial parietal cortex. $\boldsymbol{b}$, Same convention as in Figure 4 with the exception that the histograms are based on phase estimates of alpha activity in the left and right parietal cortex (top) and left thalamus (bottom).

Table 3. MNI coordinates for reversed TC-PAC

\begin{tabular}{|c|c|c|c|}
\hline \multirow[b]{2}{*}{ Anatomical label } & \multicolumn{3}{|c|}{ MNI coordinates } \\
\hline & $x$ & $y$ & $z$ \\
\hline Left precuneus & -22 & -68 & 42 \\
\hline Right precuneus & 20 & -42 & 32 \\
\hline Left thalamus & -14 & -30 & 0 \\
\hline Left thalamus & -10 & -26 & 2 \\
\hline
\end{tabular}

age. The results show that (1) thalamic activity is not related to spurious signal leakage from cortical sources and that (2) TC interactions can be reconstructed from seed regions in both thalamic and parietal regions, which further validates the robustness of the reconstructed TC-interactions.

\section{Phasic inhibition and default mode network}

Our data are in line with recent experimental studies and theoretical models of TC alpha oscillations, which highlight the impact of thalamic activity on neocortical networks (Lorincz et al., 2009; Saalmann et al., 2012; Vijayan and Kopell, 2012). Specifically, data from electrophysiological in vitro recordings and computational models of TC circuits show that during strong phasic inhibition the spiking of relay-mode neurons and pyramidal cells are entrained by the phase of the alpha cycle (Lorincz et al., 2009; Vijayan and Kopell, 2012). In agreement with previous findings, which have related alpha oscillations with fluctuations of cortical excitability (Lorincz et al., 2009; Dugué et al., 2011; Haegens et al., 2011; Scheeringa et al., 2011) and broadband gamma-power with neuronal spiking (Rasch et al., 2008; Manning et al., 2009; Whittingstall and Logothetis, 2009; Buzsáki et al., 2012), one possible
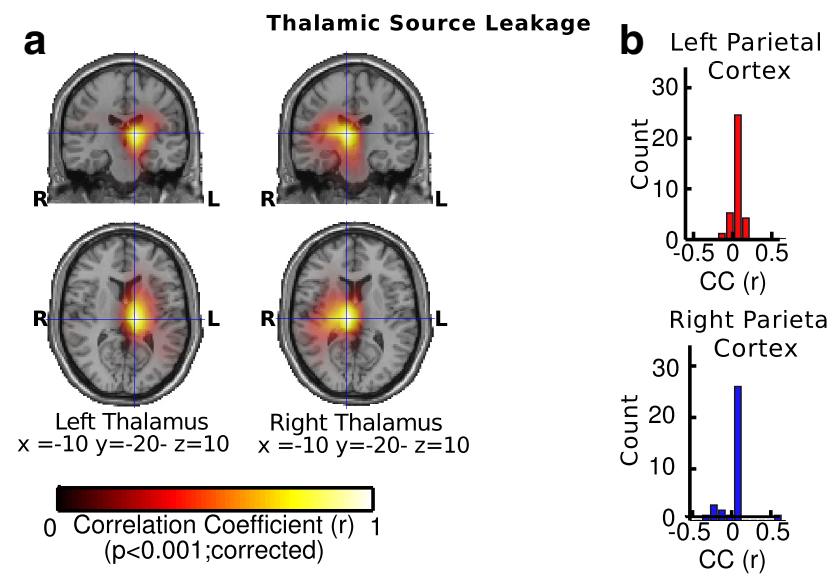

Figure 6. Thalamic source leakage. $\boldsymbol{a}$, Source maps of pearson correlation coefficients (CC) for the correlation of spatial filters in the left and right thalamus and spatial filters at brain-wide source locations (left). $\boldsymbol{b}$, Histograms showing the distribution of pearson CCs across participants for the correlation of spatial filters in the left and right thalamus and spatial filters in the left and right posterior-medial parietal cortex.

implication of our data is that the modulation of cortical broadband gamma activity reflects phasic inhibition of neuronal activity in TC networks.

From this perspective, our results may indicate that the thalamus exerts a modulatory influence on the posterior medial parietal cortex, a brain region that has important anatomical connections with the dorsal part and the higher associative nuclei of the thalamus (Morgane et al., 2005; Cavanna and Trimble, 2006) and which forms a central hub in the default mode network (Gusnard et al., 2001; 
Raichle et al., 2001; Raichle and Snyder, 2007). This hypothesis is supported by functional MRI (fMRI) and combined electroencephalographic/fMRI studies, which demonstrate that during the eyesclosed resting state alpha power in the visual cortex is associated with a reduced functional connectivity between the visual cortex and the thalamus (Scheeringa et al., 2012), whereas functional connectivity between the thalamus and the posterior medial parietal cortex is enhanced (Gur et al., 1995). Similarly, our data show that alpha power in the visual cortex was enhanced as compared with alpha power in the posterior medial parietal cortex, thereby suggesting that the difference in alpha power could reflect a reduction in functional connectivity between visual areas and the thalamus during the absence of visual input.

\section{Methodological implications}

The current data highlight the possibility that MEG may be suitable to identify modulatory interactions within TC-networks. However, because magnetic fields decay rapidly with increasing distance, source localization of subcortical activity in MEG recordings remains challenging. So far, most electrophysiological experiments in humans involving subcortical structures are conducted in surgical patients (Cohen et al., 2009; Axmacher et al., 2010; Staudigl et al., 2012; Fitzgerald et al., 2013). A limited number of MEG studies, nevertheless, have reported on thalamic and other subcortical sources (Ribary et al., 1991; Tesche, 1996a,b; Gross et al., 2001; Bish et al., 2004, Jerbi et al., 2007; Lou et al., 2010; Poch et al., 2011; Balderston et al., 2013).

The present study suggests a relationship between the phase of thalamic alpha and cortical gamma-band activity in MEG-recorded resting-state networks. Importantly, our main finding of a modulation of gamma-band power in parietal cortex through the phase of alpha-band oscillations in thalamus is supported by the close correspondence between TC-conduction latencies in our data and previous evidence from electrophysiological recordings (Lopes da Silva et al., 1980). Furthermore, anatomical studies have shown that several nuclei, such as the LGN and the thalamic reticular nucleus are composed of pyramidal cells, which have a longitudinal (anterior-posterior) dendritic architecture (Yelnik et al., 1984) that could contribute to the generation of tangential fields, which are measured by MEG (Ahlfors et al., 2010). In agreement with these findings, stimulation studies indicate that thalamic fields may achieve up to $21 \%$ ( $25 \mathrm{fT}$ ) of the total field strength produced by cortical sources ( $120 \mathrm{fT}$; Attal et al., 2007). Crucially, Attal et al. (2007) demonstrated that thalamic generators of oscillatory activity at alpha frequencies can be identified from $30 \mathrm{~s}$ long segments of eyes-closed resting-state recordings.

Moreover, recent modeling studies support this possibility through highlighting that MEG signals are particularly sensitive to rhythmic activity generated in the TC core pathway as compared with the TC matrix pathway (Bonjean et al., 2012). The TC core pathway connects the principal sensory nuclei as well as the pulvinar with the cortex through densely organized afferents fibers, which terminate in specifically localized zones (deep layers III and layer IV; Jones, 2002). Accordingly, one possible interpretation is that either the LGN or the pulvinar contribute to modulate gamma-band activity in the posterior medial parietal cortex through coherent alpha oscillations via the TC core pathway. This interpretation is supported by our observation of enhanced phase synchronization between the thalamus and posterior medial parietal regions, suggesting that coherent alpha generators in both regions contribute to the modulation of gamma-band activity in the posterior medial parietal cortex.

One limitation of the present study is the spatial resolution of the MEG method, which is in the centimeter range and therefore does not allow inferences about specific thalamic nuclei. Moreover, the left lateralization of thalamic MI values revealed by the reversed-seed region analysis may reflect the activity of a merged thalamic source as beamforming will tend to merge two spatially separated sources into a single source if the geometrical distance between both sources is $<3 \mathrm{~cm}$ (Van Veen et al., 1997).

\section{Summary}

Despite these limitations, the current study provides a first step towards the noninvasive detection of TC interactions with MEG. To further validate the present findings, further studies are required using invasive electrophysiological recordings to identify the role of thalamic nuclei such as the LGN (Lorincz et al., 2009) and the pulvinar (Lopes da Silva et al., 1980; Saalmann et al., 2012) in the generation of alpha oscillations and the modulation of cortical gamma-band activity.

The thalamus forms densely connected networks with many neocortical regions (Sherman and Guillery, 2000; Saalmann and Kastner, 2009). Source imaging of the thalamus with noninvasive techniques may thus provide important insights into the role of the thalamus in the context of cognitive tasks. Furthermore, if MEG proves to be capable of monitoring thalamic activity, it may have important clinical implications, as TC dysfunctions have been centrally involved in the pathophysiology of several severe psychiatric disorders, such as schizophrenia (O'Donnell and Grace, 1998; Pinault, 2011).

\section{References}

Adrian ED, Matthews BHC (1934) The Berger rhythm: potential changes from the occipital lobe in man. Brain 57:355-385.

Ahlfors SP, Han J, Belliveau JW, Hämäläinen MS (2010) Sensitivity of MEG and EEG to source orientation. Brain Topogr 23:227-232. CrossRef Medline

Andersen P, Andersson SA (1968) Physiological basis of the alpha rhythm., Appleton-Century-Crofts. New York.

Attal Y, Bhattacharjee M, Yelnik J, Cottereau B, Lefevre J, Okada Y, Bardinet E, Chupin M, Baillet S (2007) Modeling and detecting deep brain activity with MEG and EEG. Conf Proc IEEE Eng Med Biol Soc 2007:4937-4940. Medline

Axmacher N, Henseler MM, Jensen O, Weinreich I, Elger CE, Fell J (2010) Cross-frequency coupling supports multi-item working memory in the human hippocampus. Proc Natl Acad Sci U S A 107:3228-3233. CrossRef Medline

Balderston NL, Schultz DH, Baillet S, Helmstetter FJ (2013) How to detect amygdala activity with magnetoencephalography using source imaging. J Vis Exp 76:50212. CrossRef Medline

Bell AJ, Sejnowski TJ (1995) An information-maximization approach to blind separation and blind deconvolution. Neural Comput 7:1129-1159. CrossRef Medline

Berger H (1929) Über das Elektroenkephalogramm des Menschen. Archiv für Psychiatrie und Nervenkrankheiten 87: 527:570.

Bish JP, Martin T, Houck J, Ilmoniemi RJ, Tesche C (2004) Phase shift detection in thalamocortical oscillations using magnetoencephalography in humans. Neurosci Lett 362:48-52. CrossRef Medline

Bollimunta A, Chen Y, Schroeder CE, Ding M (2008) Neuronal mechanisms of cortical alpha oscillations in awake-behaving macaques. J Neurosci 28:9976-9988. CrossRef Medline

Bollimunta A, Mo J, Schroeder CE, Ding M (2011) Neuronal mechanisms and attentional modulation of corticothalamic $\alpha$ oscillations. J Neurosci 31:4935-4943. CrossRef Medline

Bonjean M, Baker T, Bazhenov M, Cash S, Halgren E, Sejnowski T (2012) Interactions between core and matrix thalamocortical projections in human sleep spindle synchronization. J Neurosci 32:5250-5263. CrossRef Medline

Buzsáki G, Anastassiou CA, Koch C (2012) The origin of extracellular fields and currents-EEG, ECoG, LFP and spikes. Nat Rev Neurosci 13:407-420. CrossRef Medline

Cavanna AE, Trimble MR (2006) The precuneus: a review of its functional anatomy and behavioural correlates. Brain 129:564-583. CrossRef Medline

Chorlian DB, Porjesz B, Begleiter H (2006) Amplitude modulation of gamma band oscillations at alpha frequency produced by photic driving. Int J Psychophysiol 61:262-278. CrossRef Medline 
Cohen MX, Axmacher N, Lenartz D, Elger CE, Sturm V, Schlaepfer TE (2009) Good vibrations: cross-frequency coupling in the human nucleus accumbens during reward processing. J Cogn Neurosci 21:875-889. CrossRef Medline

da Silva FH, van Lierop TH, Schrijer CF, van Leeuwen WS (1973) Organization of thalamic and cortical alpha rhythms: spectra and coherences. Electroencephalogr Clin Neurophysiol 35:627-639. CrossRef Medline

Dugué L, Marque P, VanRullen R (2011) The phase of ongoing oscillations mediates the causal relation between brain excitation and visual perception. J Neurosci 31:11889-11893. CrossRef Medline

Fitzgerald TH, Valentin A, Selway R, Richardson MP (2013) Crossfrequency coupling within and between the human thalamus and neocortex. Front Hum Neurosci 7:84. Medline

Foster BL, Parvizi J (2012) Resting oscillations and cross-frequency coupling in the human posteromedial cortex. Neuroimage 60:384-391. CrossRef Medline

Gross J, Kujala J, Hamalainen M, Timmermann L, Schnitzler A, Salmelin R (2001) Dynamic imaging of coherent sources: studying neural interactions in the human brain. Proc Natl Acad Sci U S A 98:694-699. CrossRef Medline

Gur RC, Mozley LH, Mozley PD, Resnick SM, Karp JS, Alavi A, Arnold SE, Gur RE (1995) Sex differences in regional cerebral glucose metabolism during a resting state. Science 267:528-531. CrossRef Medline

Gusnard DA, Raichle ME, Raichle ME (2001) Searching for a baseline: functional imaging and the resting human brain. Nat Rev Neurosci 2:685694. CrossRef Medline

Haegens S, Nácher V, Luna R, Romo R, Jensen O (2011) $\alpha$-Oscillations in the monkey sensorimotor network influence discrimination performance by rhythmical inhibition of neuronal spiking. Proc Natl Acad Sci U S A 108:19377-19382. CrossRef Medline

Hughes SW, Crunelli V (2005) Thalamic mechanisms of EEG alpha rhythms and their pathological implications. Neuroscientist 11:357-372. CrossRef Medline

Jahnsen H, Llinás R (1984) Ionic basis for the electro-responsiveness and oscillatory properties of guinea-pig thalamic neurones in vitro. J Physiol 349:227-247. Medline

Jensen O, Mazaheri A (2010) Shaping functional architecture by oscillatory alpha activity: gating by inhibition. Front Hum Neurosci 4:186. Medline

Jensen O, Bonnefond M, VanRullen R (2012) An oscillatory mechanism for prioritizing salient unattended stimuli. Trends Cogn Sci 16:200-206. CrossRef Medline

Jerbi K, Lachaux JP, N'Diaye K, Pantazis D, Leahy RM, Garnero L, Baillet S (2007) Coherent neural representation of hand speed in humans revealed by MEG imaging. Proc Natl Acad Sci U S A 104:7676-7681. CrossRef Medline

Jones EG (2002) Thalamic circuitry and thalamocortical synchrony. Philos Trans R Soc Lond B Biol Sci 357:1659-1673. CrossRef Medline

Klimesch W, Sauseng P, Hanslmayr S (2007) EEG alpha oscillations: the inhibition-timing hypothesis. Brain Res Rev 53:63-88. CrossRef Medline

Kraskov A, Stögbauer H, Grassberger P (2004) Estimating mutual information. Phys Rev E Stat Nonlin Soft Matter Phys 69:066138. CrossRef Medline

Kullback S, Leibler RA (1951) On information and sufficiency. Ann Math Stat 22:79-86. CrossRef

Lachaux JP, Rodriguez E, Martinerie J, Varela FJ (1999) Measuring phase synchrony in brain signals. Hum Brain Mapp 8:194-208. CrossRef Medline

Lindner M, Vicente R, Priesemann V, Wibral M (2011) TRENTOOL: a Matlab open source toolbox to analyse information flow in time series data with transfer entropy. BMC Neurosci 12:119. CrossRef Medline

Lopes da Silva FH, Vos JE, Mooibroek J, Van Rotterdam A (1980) Relative contributions of intracortical and thalamo-cortical processes in the generation of alpha rhythms, revealed by partial coherence analysis. Electroencephalogr Clin Neurophysiol 50:449-456. CrossRef Medline

Lorincz ML, Kékesi KA, Juhász G, Crunelli V, Hughes SW (2009) Temporal framing of thalamic relay-mode firing by phasic inhibition during the alpha rhythm. Neuron 63:683-696. CrossRef Medline

Lou HC, Gross J, Biermann-Ruben K, Kjaer TW, Schnitzler A (2010) Coherence in consciousness: paralimbic gamma synchrony of self-reference links conscious experiences. Hum Brain Mapp 31:185-192. Medline

Manning JR, Jacobs J, Fried I, Kahana MJ (2009) Broadband shifts in local field potential power spectra are correlated with single-neuron spiking in humans. J Neurosci 29:13613-13620. CrossRef Medline
Maris E, Oostenveld R (2007) Nonparametric statistical testing of EEG- and MEG-data. J Neurosci Methods 164:177-190. CrossRef Medline

Miller KJ (2010) Broadband spectral change: evidence for a macroscale correlate of population firing rate? J Neurosci 30:6477-6479. CrossRef Medline

Miller KJ, Hermes D, Honey CJ, Sharma M, Rao RP, den Nijs M, Fetz EE, Sejnowski TJ, Hebb AO, Ojemann JG, Makeig S, Leuthardt EC (2010) Dynamic modulation of local population activity by rhythm phase in human occipital cortex during a visual search task. Front Hum Neurosci 4:197. Medline

Morgane PJ, Galler JR, Mokler DJ (2005) A review of systems and networks of the limbic forebrain/limbic midbrain. Prog Neurobiol 75:143-160. CrossRef Medline

Nolte G (2003) The magnetic lead field theorem in the quasi-static approximation and its use for magnetoencephalography forward calculation in realistic volume conductors. Phys Med Biol 48:3637-3652. CrossRef Medline

O'Donnell P, Grace AA (1998) Dysfunctions in multiple interrelated systems as the neurobiological bases of schizophrenic symptom clusters. Schizophr Bull 24:267-283. CrossRef Medline

Oostenveld R, Fries P, Maris E, Schoffelen JM (2011) FieldTrip: open source software for advanced analysis of MEG, EEG, and invasive electrophysiological data. Comput Intell Neurosci 2011:156869. Medline

Osipova D, Hermes D, Jensen O (2008) Gamma power is phase-locked to posterior alpha activity. PLoS One 3:e3990. CrossRef Medline

Percival DB, Walden AT (1998) Spectral analysis for physical applications: multitaper and conventional univariate techniques. Cambridge, MA: Cambridge UP.

Pinault D (2011) Dysfunctional thalamus-related networks in schizophrenia. Schizophr Bull 37:238-243. CrossRef Medline

Poch C, Fuentemilla L, Barnes GR, Düzel E (2011) Hippocampal thetaphase modulation of replay correlates with configural-relational shortterm memory performance. J Neurosci 31:7038-7042. CrossRef Medline

Ragwitz M, Kantz H (2002) Markov models from data by simple nonlinear time series predictors in delay embedding spaces. Phys Rev E Stat Nonlin Soft Matter Phys 65:056201. CrossRef Medline

Raichle ME, Snyder AZ (2007) A default mode of brain function: a brief history of an evolving idea. Neuroimage 37:1083-1090; discussion 10971099. CrossRef Medline

Raichle ME, MacLeod AM, Snyder AZ, Powers WJ, Gusnard DA, Shulman GL (2001) A default mode of brain function. Proc Natl Acad Sci U S A 98:676-682. CrossRef Medline

Rasch MJ, Gretton A, Murayama Y, Maass W, Logothetis NK (2008) Inferring spike trains from local field potentials. J Neurophysiol 99:1461-1476. CrossRef Medline

Ribary U, Ioannides AA, Singh KD, Hasson R, Bolton JP, Lado F, Mogilner A, Llinás R (1991) Magnetic field tomography of coherent thalamocortical 40-Hz oscillations in humans. Proc Natl Acad Sci U S A 88:11037-11041. CrossRef Medline

Saalmann YB, Kastner S (2009) Gain control in the visual thalamus during perception and cognition. Curr Opin Neurobiol 19:408-414. CrossRef Medline

Saalmann YB, Pinsk MA, Wang L, Li X, Kastner S (2012) The pulvinar regulates information transmission between cortical areas based on attention demands. Science 337:753-756. CrossRef Medline

Sauseng P, Klimesch W, Heise KF, Gruber WR, Holz E, Karim AA, Glennon M, Gerloff C, Birbaumer N, Hummel FC (2009) Brain oscillatory substrates of visual short-term memory capacity. Curr Biol 19:1846-1852. CrossRef Medline

Scheeringa R, Mazaheri A, Bojak I, Norris DG, Kleinschmidt A (2011) Modulation of visually evoked cortical FMRI responses by phase of ongoing occipital alpha oscillations. J Neurosci 31:3813-3820. CrossRef Medline

Scheeringa R, Petersson KM, Kleinschmidt A, Jensen O, Bastiaansen MC (2012) EEG alpha power modulation of fMRI resting state connectivity. Brain Connect 2:254-264. CrossRef Medline

Sekihara K, Nagarajan SS, Poeppel D, Marantz A (2004) Asymptotic SNR of scalar and vector minimum-variance beamformers for neuromagnetic source reconstruction. IEEE Trans Biomed Eng 51:1726-1734. CrossRef Medline

Sherman SM, Guillery RW (2000) Exploring the thalamus, Ed 1. San Diego: Academic.

Spaak E, Bonnefond M, Maier A, Leopold DA, Jensen O (2012) Layer- 
specific entrainment of gamma-band neural activity by the alpha rhythm in monkey visual cortex. Curr Biol 22:2313-2318. CrossRef Medline

Staudigl T, Zaehle T, Voges J, Hanslmayr S, Esslinger C, Hinrichs H, Schmitt FC, Heinze HJ, Richardson-Klavehn A (2012) Memory signals from the thalamus: early thalamocortical phase synchronization entrains gamma oscillations during long-term memory retrieval. Neuropsychologia 50: 3519-3527. CrossRef Medline

Tallon-Baudry C, Bertrand O (1999) Oscillatory gamma activity in humans and its role in object representation. Trends Cogn Sci 3:151-162. CrossRef Medline

Tarkiainen A, Liljeström M, Seppä M, Salmelin R (2003) The 3D topography of MEG source localization accuracy: effects of conductor model and noise. Clin Neurophysiol 114:1977-1992. CrossRef Medline

Tesche CD (1996a) MEG imaging of neuronal population dynamics in the human thalamus. Electroencephalogr Clin Neurophysiol [Suppl 47]:8190. Medline

Tesche CD (1996b) Non-invasive imaging of neuronal population dynamics in human thalamus. Brain Res 729:253-258. CrossRef Medline

Tort AB, Komorowski R, Eichenbaum H, Kopell N (2010) Measuring phase-amplitude coupling between neuronal oscillations of different frequencies. J Neurophysiol 104:1195-1210. CrossRef Medline

Van Veen BD, van Drongelen W, Yuchtman M, Suzuki A (1997) Localization of brain electrical activity via linearly constrained minimum variance spatial filtering. IEEE Trans Biomed Eng 44:867-880. CrossRef Medline

VanRullen R, Koch C (2003) Is perception discrete or continuous? Trends Cogn Sci 7:207-213. CrossRef Medline
Varela FJ, Toro A, John ER, Schwartz EL (1981) Perceptual framing and cortical alpha rhythm. Neuropsychologia 19:675-686. CrossRef Medline

Vicente R, Wibral M, Lindner M, Pipa G (2011) Transfer entropy-a modelfree measure of effective connectivity for the neurosciences. J Comput Neurosci 30:45-67. CrossRef Medline

Vijayan S, Kopell NJ (2012) Thalamic model of awake alpha oscillations and implications for stimulus processing. Proc Natl Acad Sci U S A 109: 18553-18558. CrossRef Medline

Voytek B, Canolty RT, Shestyuk A, Crone NE, Parvizi J, Knight RT (2010) Shifts in gamma phase-amplitude coupling frequency from theta to alpha over posterior cortex during visual tasks. Front Hum Neurosci 4:191. Medline

Whittingstall K, Logothetis NK (2009) Frequency-band coupling in surface EEG reflects spiking activity in monkey visual cortex. Neuron 64:281-289. CrossRef Medline

Wibral M, Pampu N, Priesemann V, Siebenhühner F, Seiwert H, Lindner M, Lizier JT, Vicente R (2013) Measuring information-transfer delays. PLoS One 8:e55809. CrossRef Medline

Yanagisawa T, Yamashita O, Hirata M, Kishima H, Saitoh Y, Goto T, Yoshimine T, Kamitani Y (2012) Regulation of motor representation by phase-amplitude coupling in the sensorimotor cortex. J Neurosci 32: 15467-15475. CrossRef Medline

Yelnik J, Percheron G, François C (1984) A Golgi analysis of the primate globus pallidus. II. Quantitative morphology and spatial orientation of dendritic arborizations. J Comp Neurol 227:200-213. CrossRef Medline 